\title{
Efectos Regionales de la Crisis Financiera para los Sistemas Bancarios Brasileños y Españoles*
}

\section{Regional Effects of the Financial Crisis for Brazilian and Spanish Bank Systems}

\author{
Fernanda Faria Silva* \\ Marco Crocco** \\ Fabiana Borges Teixeira dos Santos*** \\ Marco Antônio Duarte Filho****
}

\begin{abstract}
Resumen: El objetivo de este artículo es presentar un análisis comparativo de los efectos regionales de la última crisis financiera en los sistemas bancarios brasileño y español. Se identificaron las características de la banca en los dos países y se estableció que en el caso de Brasil todavía persiste una concentración del crédito en las regiones de mayor desarrollo económico, así como mayor preferencia por la liquidez en las regiones periféricas. La crisis financiera ha acentuado este padrón. En el caso español, se esperaba que la segmentación del mercado (con la presencia de los bancos regionales) contribuyera a la mitigación de los efectos de la crisis. Sin embargo, el proceso de reestructuración bancaria ha provocado cambios en el mapa bancario español. El gran número de fusiones, adquisiciones y operaciones ha ampliado la concentración del mercado, reduciendo el crédito en las regiones periféricas, lo que acentúa las disparidades regionales.
\end{abstract}

Palabras clave: Sistema bancario. Crisis financiera. Crédito. Suporte financiero.

Abstract: The aim of this article is to present an exploratory and comparative analysis of the regional effects of the last financial crisis in the Brazilian and Spanish banking systems. Recent banking characteristics of the Brazilian and Spanish banking systems were identified. In the first case, the evidence of credit concentration in more economically developed regions is dominant and increased liquidity preference in peripherical regions. The last crisis has accentuated this pattern. In the Spanish case, the market segmentation (with the presence of regional banks) mitigated the effects of the crisis. However, the recent process of bank restructuring has caused modifications in the Spanish banking map. The large number of mergers, acquisitions and interventions

* Los autores agradecen a la Fundação de Amparo à Pesquisa do Estado de Minas Gerais (Fapemig) por el suporte financiero.

* $\quad$ Profesora doctora de la Universidad Federal de Ouro Preto (UFOP) y Laboratorio de Estudios en Moneda y Territorio (LEMTe) de la Universidade Federal de Minas Gerais (UFMG). E-mail: fernfaria@gmail.com

** Profesor titular del Centro de Desarollo y Planeamento Regional de la UFMG. Coordinador del LEMTe (Cedeplar/UFMG). E-mail: crocco@ cedeplar.ufmg.br

*** Investigadora y coordinadora del LEMTe (Cedeplar/UFMG). E-mail: fabiana@cedeplar.ufmg.br

**** Master en Economía por la Fundação Getúlio Vargas (FGV-SP). E-mail: maduartefilho@gmail.com 
have increased the market concentration, reduced the credit in peripheral regions, strengthening the regional inequalities.

Keywords: Banking system. Financial crisis. Credit.

JEL Classification: G01; G20; G34; R10; R50.

\section{Introducción}

Diversas interpretaciones de la reciente crisis financiera en la economía y las finanzas apuntan para la mala asignación y a la cantidad excesiva del crédito concedido sin una evaluación a fondo de los valores negociados en el mercado financiero. El sistema bancario comenzó a realizar sus operaciones a través de productos estructurados y derivados, multiplicando los riesgos de impago y de apalancamiento. La mayor desregulación de los mercados y la expansión de los tipos y volúmenes de las innovaciones financieras han potencializado la estrategia de expansión de los créditos de alto riesgo, permitiendo ampliar la rentabilidad de los bancos.

Las transacciones complejas entre el sistema bancario y los miembros del "shadow banking system" han aumentado los riesgos globales en el mercado financiero y ha obstaculizado las aplicaciones de las normas reglamentarias. La transferencia del riesgo de los balances de los bancos a otras instituciones financieras no bancarias, así como la expansión de los préstamos subprime fue potenciada por las innovaciones en productos y servicios financieros. Evidentemente, esta nueva dinámica ha contribuido para el aumento de la disparidad entre la riqueza productiva y financiera, sobresaliendo esta última. La lógica financiera comenzó a influir en las decisiones de inversión en el sector real de la economía, profundizando el proceso de financiarizacion. Resulta de este proceso que las operaciones financieras se han vuelto más especulativas. Además, otra consecuencia importante es la pérdida de la "funcionalidad del sistema bancario" cuyo principal impacto es la mayor restricción de la oferta de crédito a largo plazo (REZENDE, 2012; HERMANN; PAULA, 2011; CROCCO, 2010). Teniendo en cuenta las implicaciones tomadas por la reciente crisis financiera, es necesario examinar algunos de sus impactos regionales en el sistema bancario, más concretamente, en los casos de Brasil y España.

Este artículo se justifica tanto por el alcance de la última crisis en los dos países, como también porque la mayor parte de los estudios en la literatura económica aún no han incorporado el enfoque regionalizado en sus análisis. Los dos países fueron elegidos por presentaren algunas características similares, sobre todo por el tipo de desarrollo de sus sistemas financieros (notoriamente bank based, o sea, con mayor participación del sector bancario y más baja para el mercado de capitales). Además, son dos economías con grandes diferencias en sus desarrollos regionales y que también se pueden considerar periferias (Brasil, en el ámbito mundial e 
España en términos europeos). Entre las principales características distintivas se puede considerar: las diferencias institucionales (regímenes monetarios, las cuestiones salariales, las relaciones con el Estado, el nivel de integración internacional), la segmentación del mercado bancario, así como las diferencias en las estructuras productivas y regulatorias que teóricamente podrían contribuir a una mayor mitigación de los efectos de la crisis en las regiones menos desarrolladas.

La hipótesis central de este artículo es que en las regiones periféricas de ambos los países, los efectos de la reciente crisis financiera deberá ser más pronunciado debido a las limitaciones estructurales de estas regiones, a la menor oferta de financiamiento y por la mayor preferencia por la liquidez de los agentes (bancos y demandantes de fondos). En Brasil, la presencia mayoritaria de los bancos (públicos y privados) que operan a escala nacional y el reducido número de bancos regionales son factores que contribuyen para que la preferencia por la liquidez sea mayor en las regiones periféricas, lo que afecta a la disponibilidad de recursos y dificulta la articulación de acciones y estrategias de estas instituciones para el desarrollo local.

En el caso español, en teoría, la presencia de bancos regionales facilitaría la provisión de recursos a las regiones más vulnerables y con mayor peso de actividades como la agricultura, la ganadería y el sector inmobiliario, de gran importancia económica para las regiones más deprimidas. Sin embargo, la reciente crisis financiera ha provocado grandes cambios en el mapa bancario español. Los efectos se verán en el medio y largo plazo con el fin del proceso de reestructuración de los bancos, concretado en el último noviembre. Se teme que una de las consecuencias más graves caerá sobre la pérdida de funcionalidad del sistema bancario para el desarrollo de las regiones (en especial, las periféricas).

A pesar de las crecientes innovaciones financieras en el mercado, el mayor número de activos financieros no está directamente asociado con una mayor oferta de crédito a las regiones económicamente menos dinámicas, lo que hace más restricta la oferta de financiación para estas regiones, tanto en términos de volumen como de las fuentes de recursos (SILVA, 2011; CROCCO; FIGUEIREDO; SANTOS, 2010). Cabe señalar que la reciente crisis financiera ha acentuado este patrón en ambos países, así como la concentración bancaria en las regiones económicamente más fuertes, lo que se confirma en la segunda parte de este artículo.

La estructura del trabajo consta, además de este primer apartado de carácter introductorio, de otros tres apartados. En el primer se describen los principales efectos de la reciente crisis financiera y sus repercusiones en los sistemas bancarios brasileño y español. En el segundo se hace un análisis exploratorio de los indicadores que identifican las características de los sistemas bancarios de ambos los países. Por último, se recogen a algunas conclusiones, así como algunas indicaciones sobre las líneas abiertas en este campo de investigación. 


\section{Cambios y Caracterización reciente de los Sistemas Bancarios Brasileño y Español}

\subsection{Los Efectos de la Crisis Financiera en el Sistema Bancario Brasileño}

El sector bancario brasileño ha sufrido varios cambios a partir de la década de 1990, particularmente debido al proceso de liberalización financiera. Este proceso ha ejercido varios tipos de efectos en este sector, tales como la ampliación de la competencia, una gran ola de concentración a través de fusiones y adquisiciones - inicialmente marcada por la mayor entrada de los bancos extranjeros - y la mayor concentración espacial del crédito. Las regiones periféricas eran servidas por líneas de crédito ofrecidas principalmente por bancos regionales que se han vuelto cada vez más escasos. Este factor, asociado a las propias preferencias de las instituciones bancarias ha ampliado el racionamiento de crédito para estas regiones.

Esta dinámica es resultado de las distintas estrategias bancarias en el espacio, como se muestra en la segunda sección con el análisis de los indicadores y figuras. Aunque la configuración del sistema bancario brasileño se caracterice por la presencia de grandes bancos con operaciones en todo el país, estas instituciones tienden a transferir los fondos (depósitos) de las regiones periféricas y dirigirlos hacia las centrales, que ofrecen una gama más amplia de aplicaciones y menor riesgo (CROCCO; FIGUEIREDO; SANTOS, 2010, CROCCO; CAVALCANTE; BARRA, 2005).

El final del período inflacionario, como resultado de las medidas aplicadas con el Plan Real y la fuerte caída de los ingresos por la pérdida del floating, los bancos brasileños pasaron a revisar sus estrategias e sus inversiones de cartera. Desde entonces, ha habido un aumento significativo en las operaciones de crédito en el activo de los balances bancarios como mecanismo de ampliación de los ingresos por medio de los préstamos.

Sin embargo, las sucesivas crisis de la década de 1990 (México, Asia, Rusia) aumentó la percepción del efecto contagio en las economías emergentes, lo que hice con que las autoridades monetarias adoptasen una política monetaria más restrictiva, en especial, utilizando de altos tipos de interés con el fin de evitar a la salida y mismo atraer capital extranjero. Los bancos pasaran a adquirir bonos - un tipo de inversión financiera más rentable y menos arriesgada do que los préstamos - lo que ha culminado en una importante fuente de ingresos. El aumento de las operaciones con bonos ha favorecido el resurgimiento del componente especulativo de la economía debido a la posibilidad de ganar con arbitraje. El aumento de rendimiento de los bonos se ha convertido en un importante incentivo para este 
tipo de operaciones y el movimiento de estos ingresos ha seguido una trayectoria de alta de los tipos de interés reales (Selic) hasta el año de 2008.

A mediados de 2009, con el cambio de escenario económico internacional y la nueva orientación de la política macroeconómica, el Banco Central de Brasil $(\mathrm{BCB})$ ha pasado a reducir de modo gradual a los tipos de interés. Con la inestabilidad financiera generada por la última crisis financiera y la depreciación del dólar en este año, los ingresos por bonos vinculados a la tasa de cambio también se comprimieron. En el segundo mandato del gobierno Lula, el apoyo al segmento de crédito se consolidó con el estímulo a los préstamos, que se ha vuelto una fuente importante de ingresos para los bancos. Además, se ha aumentado también la proporción de los ingresos referentes a los servicios bancarios debido a las actividades bancarias no incluidos en el balance, tales como algunos tipos de titulización y derivados. Cabe señalar que la búsqueda de una mayor rentabilidad por parte de los bancos hace con que aumente la participación de activos más líquidos. En respuesta al mayor riesgo de liquidez se ha ampliado los márgenes de seguridad bancarios y exigencias de altas garantías, lo que refleja la contracción del crédito, especialmente para las operaciones a largo plazo.

En esta última crisis financiera, el impacto en el sistema bancario brasileño fue reducido no sólo por la fuerza de los grandes bancos privados nacionales que todavía estaban poco apalancados, sino también por las medidas anti cíclicas adoptadas por el Gobierno Lula. Los bancos brasileños estaban menos expuestos a las operaciones de secularización y a los derivados vinculados a las hipotecas de alto riesgo. Kregel (2009) explica que los altos tipos de interés garantizaban alta rentabilidad a los bancos brasileños, por esto estaban menos propensos a las actividades de alto riesgo. Así, los bancos brasileños fueron capaces de mantener altas tasas de rentabilidad - incluso en el contexto de crisis - superiores a los observados en los países desarrollados y otros países emergentes.

Por el contrario, en los mercados financieros de los países desarrollados, la menor rentabilidad de los bancos comerciales y de inversión asociados con los tipos de interés relativamente bajos (e incluso negativos) fueron esenciales para la expansión del mercado de titulización y derivados de crédito.

Sin embargo, aunque los bancos brasileños fueron ligeramente contaminados con las operaciones estructuradas no significa que la crisis financiera no ha requerido importantes esfuerzos de parte del Banco Central y, por lo tanto, que no ha provocado efectos importantes en los lados reales y financieros de la economía. Mismo sin tener una corrida bancaria, el Banco Central brasileño intervino en la provisión de liquidez en moneda local. ${ }^{1}$

1 Las principales medidas para garantizar mayor liquidez en la economía y la solidez del sistema bancario fueron las intervenciones en el Fondo de Garantía de Crédito y en la política de redescuento. En marzo de 2009, se han intensificado los depósitos en poder del Fondo de Garantía de Garantía de Crédito (FGC), con el fin de garantizar la recuperación de los bancos de pequeño y mediano portes. 
El aumento de la tasa Selic se ha invertido en enero de 2009, cuando la depreciación de la moneda "Real" ya no imponía riesgos a la economía. Posteriormente fue estimulada la expansión de la oferta de crédito por parte de los bancos públicos: la "Caixa Econômica Federal", el Banco de Brasil y el Banco Nacional de Desarrollo Económico y Social (BNDES). Estas instituciones tuvieron una fuerte actuación anticíclica y fueron autorizados por el Banco Central para participar de las adquisiciones de activos de los bancos con problemas de endeudamiento o de liquidez, ampliando la cuota de mercado de los bancos públicos.

Como Brasil ha presentado un crecimiento significativo a partir de 2007, la demanda por crédito por parte de las empresas fue expandida. Además, por el lado de la oferta, fueron ampliadas las líneas de crédito, principalmente para capital de explotación. La moneda brasileña, el Real, ha estado valorizado desde 2004, lo que hice con que varias empresas contratasen operaciones de hedge e otras de carácter más especulativo. La relativa estabilidad del cambio hice con que los bancos y las empresas subestimasen el riesgo de operaciones con derivados cambiales. Con la intensificación de la crisis financiera en 2008, el movimiento de fuga de capitales ha provocado una rápida depreciación del Real, dificultando el pago de diversos contratos (en moneda extranjera) y causando grandes problemas, principalmente a las empresas exportadoras (tales como Sadia y Aracruz), siendo necesaria, la intervención del BNDES.

Otro efecto de la crisis en Brasil fue la restricción de las líneas de crédito al exterior para el comercio. Las pérdidas con los derivados vinculados al dólar norteamericano provocaron mayor aversión al riesgo y la preferencia por la liquidez de los bancos. Según Freitas (2009), respecto a la dinámica del mercado interbancario nacional, los bancos ignoraban la exposición excesiva de los agentes a los derivados cambiarios y pasaron a restringir la oferta de crédito a las empresas y los particulares, concentrando sus operaciones en bonos públicos. El aumento de la tasa Selic ha sido el principal incentivo para este cambio de estrategia.

Con la reducción de los flujos de capital hacia las economías emergentes y aumento de la aversión al riesgo, los bancos y las pequeñas y medianas empresas, que dependen del mercado interbancario, incurrieran en serios problemas de liquidez. Por el contrario, los grandes bancos nacionales ampliaron sus bases de recursos, debido a su mayor solidez y a la actuación de modo pulverizado (con gran escala para captación de depósitos).

Sin embargo, debe ser señalado que las instituciones bancarias públicas y privadas presentan diferentes estrategias de operación. Los bancos privados tienen una dinámica más inestable en las fases del ciclo económico. En el contexto del aumento cíclico, expanden la oferta de crédito, estando más apalancados en otras operaciones. En contextos de crisis, restringen la oferta de recursos debido a la mayor preferencia por la liquidez ante a la incertidumbre. Sin embargo, históri- 
camente, los bancos públicos brasileños han tenido una actuación contra cíclica, actuando de modo más funcional, es decir, presentan una mayor capacidad de financiación a la inversión productiva, mismo en contextos de crisis.

\subsection{Caracterización reciente del Sistema Bancario Español}

Desde la década de 1980, las entidades financieras españolas han sido afectadas por las distintas medidas de desregulación con el objetivo de estimular la competencia, por la difusión de las innovaciones financieras y para la mayor difusión tecnológica. En 1987 se ha permitido la entrada de los bancos extranjeros; en 1989 fue concedida la apertura de cajas de ahorro fuera de sus territorios de origen, con la eliminación total de las restricciones para sus operaciones activas. Como consecuencias más inmediatas, se destaca el aumento relativo de la eficiencia productiva, la reducción de los precios (tipos de interés y de los márgenes de intermediación bancaria), como también el aumento de la oferta de servicios financieros y de la competitividad, principalmente por la entrada de nuevas instituciones financieras.

A pesar de la liberalización de las finanzas tener ampliado las posibilidades de intercambios financieros entre los países (especialmente entre España y el resto de la zona Euro) y mismo la apertura de nuevas oportunidades de expansión del mercado de crédito y de capital, el sector bancario sigue teniendo el mayor peso en el tejido financiero español. Así mismo, la intensificación de las fusiones y adquisiciones, características del proceso de liberalización financiera, no ha eliminado la segmentación institucional del sistema bancario español.

De este modo, se espera que el mercado bancario español tenga mejores condiciones de mitigar los efectos de la crisis en cuanto a la disponibilidad regional de crédito, debido a: i) el tipo de segmentación del mercado bancario, con una fuerte presencia de bancos regionales (cajas de ahorro) y ii) los aspectos institucionales más bien consolidados, fundamentales cuando se requiere un esfuerzo de coordinación más amplio, especialmente en contextos de crisis.

En el caso español, el sistema financiero se somete a la misma institucionalidad del Banco Central Europeo (BCE) hizo aumentar las exigencias sobre el cumplimiento de las normas establecidas para todos los países de la área monetaria común y, consecuentemente, a las medidas de austeridad firmadas en el pacto de estabilidad fiscal y monetaria. Sin embargo, estar bajo la supervisión y legislación del BCE facilita el rescate en momentos de inestabilidad, el monitoreo de las instituciones y facilita el cumplimiento de los mecanismos de enforcement.

Como aspecto positivo de la segmentación del mercado bancario español, la presencia de los bancos regionales ayudaría a reducir la preferencia por la liquidez de los agentes (los prestatarios y los prestamistas), especialmente en la provisión 
de recursos a los sectores más vulnerables de la economía. Con el proceso de reestructuración bancaria en vigor, las consecuencias solamente podrán ser evaluadas al medio y largo plazo.

Los cambios en el mapa bancario español frente a la última crisis financiera también serán destacados en este trabajo, sobre todo desde la perspectiva de los bancos regionales. Dado que estos cambios se siguen produciendo, la idea es tratar de captar algún indicio de los efectos del proceso de concentración bancaria, principalmente en lo que se refiere a la pérdida de funcionalidad para el desarrollo de las regiones españolas. Como resaltado, en el caso brasileño, la presencia mayoritaria de los bancos que operan en escala nacional y la ausencia de los bancos regionales contribuyen a que la preferencia por la liquidez de los bancos sea mayor en las regiones más periféricas, comprometiendo la disponibilidad de recursos.

Los bancos regionales son importantes para reducir la incertidumbre y por la expansión de los préstamos, ya que tienen una dinámica distinta de los bancos nacionales. La limitada disponibilidad de financiamiento es un obstáculo efectivo para la inversión y la generación de ingresos, contribuyendo a una mayor depreciación de los activos locales, más dependientes del mercado regional (DOW, 1999). En un modelo de los bancos nacionales, de acuerdo con la autora, aunque se tiene varias agencias en todo el territorio, el constante flujo de capital hacia los centros financieros ubicados en las regiones centrales tiende a reforzar las desigualdades regionales.

Por lo tanto, es importante tener en cuenta los aspectos institucionales, especialmente la necesidad de que el sistema financiero tenga un desempeño diferenciado de las regiones centrales y periféricas. La mayoría de los estudios sobre el tema defiende el punto de vista microeconómico, basado en la necesidad de la firma bancaria en obtener beneficios y asignar los recursos de manera eficiente, es decir, en la búsqueda de mayor rentabilidad en el corto plazo. Desde el punto de vista estructural, es necesario que el sistema bancario actúe de modo distinto respecto a la gestión de sus activos y direccionamiento de recursos para la financiación del desarrollo, en especial para las regiones periféricas (CARVALHO, 2007).

De modo distinto al Brasil, España cuenta con la presencia de un número de instituciones bancarias privadas regionales, tales como las cajas de ahorro y cooperativas de crédito. La desregulación financiera implementada desde 1984 hasta 2007, hizo que el número total de bancos fuera reducido desde 369 hasta 275 , gracias a las fusiones y adquisiciones.

Durante ese período, las cajas de ahorro se han reducido de 76 para 54 y las cooperativas de crédito de 149 para 83. La caída en el número de bancos comerciales fue cerca de 13,2\% (AYADI; SCHMIDT; VALVERDE, 2010). Incluso antes de este escenario, las cajas de ahorro abrieron nuevas sucursales, de lo que, 
a la diferencia de los bancos comerciales, redujeron su participación en el sistema bancario español. Estas constataciones indican una estrategia diferenciada de actuación espacial por parte de estas instituciones. En el caso de las cajas, hay una especialización en el comercio minorista, con enfoque en la proximidad territorial. Por otro lado, los bancos comerciales buscan una mayor diversificación, actuando en una mezcla de banca minorista y de inversión, combinación que altera la estructura competitiva del mercado.

La cajas de ahorro y las cooperativas de crédito tienen características que permiten identificarlos como más funcionales debido a su compromiso de ofertar recursos para sus localidades, dirigidos a los sectores y segmentos en los cuales los grandes bancos comerciales tienen mayor restricción a la financiación (tales como para las pequeñas y medianas empresas; el sector agrícola e inmobiliario; como también para áreas de exclusión geográfica y rural). Estas instituciones deben destinar un porcentaje de sus ganancias en proyectos de cohesión social (la Obra Social) o, de acuerdo con la regulación regional, garantizar la prestación de servicios financieros básicos (AYADI; SCHMIDT; VALVERDE, 2010).

Sin embargo, actualmente, todas las instituciones financieras de la Unión Monetaria Europea están sometidas a las mismas presiones para la mejora de la eficiencia y la gestión de los riesgos. Como se verá a la continuación, las cajas de ahorro están pasando por importantes transformaciones. Muchas de ellas, se han fusionado con otras cajas que presentan mayor liquidez (a través de las "fusiones frías”), o mismo con los grandes bancos comerciales, sujetándose a las mismas presiones por ampliar la competencia y la cotización de los accionistas, perdiendo su carácter funcional.

Tal escenario es problemático sobre todo si se considera que la financiación a través del mercado de capitales es aún limitado en el país, principalmente para las pequeñas y medianas empresas, mayoría en el tejido productivo español. Esta situación se agrava especialmente en las regiones más periféricas, donde el acceso a las fuentes alternativas de crédito y las instituciones formales es aún más restricto. Todos estos factores significan la grande dependencia de las firmas e agentes españolas con respecto al mercado bancario.

\subsubsection{La reciente Reestructuración del Sistema Bancario Español}

Un análisis reciente de la banca española muestra que el país experimentó un boom del crédito, especialmente entre el período de 2004 hasta 2007. Según Rodríguez-Fuentes y Silva (2010), la notable expansión del crédito bancario en España se debe, entre otros factores, a las mejoras en la eficiencia productiva del sector decurrentes de la liberalización financiera. Este proceso ha generado mayor competencia entre las instituciones y menores tipos de interés. Todavía ha puesto 
la necesidad de nuevos ajustes a las entidades financieras, a fin de que no redujesen sus market share en el segmento de los bancos minoristas tradicionales (especializada en la oferta de recursos y servicios para el comercio y las pequeñas y medianas empresas).

Inicialmente, se ha observado un aumento progresivo de los márgenes de intermediación bancaria que, teóricamente, sería compensado con niveles más altos de eficiencia, sino también por el aumento de la escala de la operación (mediante el aumento del número de operaciones de intermediación) y la exploración de nuevos mercados (a través del crecimiento de otros ingresos). El aumento en la escala de las operaciones ha sido facilitado por la existencia de una demanda creciente por recursos financieros, beneficiado por las condiciones de bajos tipos de interés. Otros factores que contribuyeron fueron las expectativas de altos rendimientos asociados a las operaciones de endeudamiento (sobre todo para el sector inmobiliario). Todas estas condiciones resultaron en el crecimiento del apalancamiento financiero, que se refleja en el excesivo endeudamiento de las familias y empresas. $^{2}$

La amplia demanda de crédito fue desfavorecida por un retroceso del escenario externo favorable, a partir de 2007. Hasta este año, los efectos de la crisis subprime no se han sentido directamente, teniendo en cuenta la baja exposición de la banca española a los productos estructurados. Desde 2008 se ha verificado la generalización de los problemas de liquidez y la crisis del sistema monetario europeo, el aumento de la desconfianza en el mercado interbancario, acentuando los impactos en el mercado financiero español.

Una reflexión importante de los impactos de la crisis financiera en España recae sobre el peso del sector inmobiliario en la economía de este país, seguido de su colapso. La crisis ha revelado el temor a la deflación de los activos y al deterioro de los balances bancarios (teniendo en cuenta la elevada participación de la banca española en este tipo de financiación). En 2009, estos problemas se hacen más evidentes debido a la mayor inestabilidad macroeconómica, seguida de la caída de la producción real y aumento del desempleo.

La necesidad de intervenir en la primera entidad financiera - la Caja Castilla La Mancha - ha significado que, a partir de entonces, se estableció un marco institucional para garantizar todas las medidas de emergencia y de estímulo económico para atenuar los efectos de la crisis. ${ }^{3}$ Por lo tanto, la insolvencia de algunas en-

2 La entrada de España a las exigencias del sistema monetario europeo tuvo como consecuencias importantes: la exigencia de una reducción sustancial de las tasas de interés en el país, tanto a fin de cumplir a los criterios de convergencia, cuanto proporcionar un acceso más inmediato y de bajo costo a los mercados financieros internacionales (debido a la disminución relativa de la preferencia por la liquidez. La expansión de la demanda de crédito como consecuencia de las expectativas futuras de revalorización de los activos inmobiliarios impulsó gran parte de la demanda de recursos y el nivel real de crecimiento del empleo en la economía española.

3 En 2009 se creó el Fondo para la Reestructuración de la Orden Bancaria (FROB), cuyo objetivo 
tidades de crédito españolas se puede entender como resultado de las estrategias de expansión en el pico del ciclo de crecimiento del crédito anterior (favorecida por una política monetaria laja), la mala gestión del riesgo pobres, agravada por la recesión económica y por la falta de financiamiento externo después del inicio de la crisis. Uno de sus principales impactos resultó en el debilitamiento del sistema bancario español, que ha requerido la aprobación del Fondo de Reestructuración de la Orden Bancaria (FROB), seguida de otras medidas, tales como el fortalecimiento de los requisitos prescritos en los acuerdos de Basilea, el aumento de los recursos propios (capital básico), reforma de la Ley de Cajas (Real Decreto-Ley 11/2010) con las disposiciones y normas para la determinación de los niveles de solvencia. ${ }^{4}$

Con la bancarización de cajas de ahorro y la presentación de los mismos a los inversores privados, se invierte el orden de prioridades. Ahora, estas entidades están sujetas a una mayor disciplina de mercado, a las técnicas de mejora de la gestión de riesgo y la búsqueda por resultados. Seguramente se verá comprometido el carácter funcional de estas instituciones. Más que el proceso de concentración bancaria y la pérdida de funcionalidad, lo que es sorprendente es la rapidez con lo que el proceso está desarrollando. Según el FMI (INTERNATIONAL MONETARY FUND, 2012), de las 50 cajas de ahorros existentes en 2009, sólo 14 llegaron a existir en 2012, siendo que la mayoría de estas instituciones se sitúan en las comunidades autónomas menos desarrolladas (como Caja Canarias, Caja Insular Canarias, Caja Jaén, Caixa Galicia, entre otros).

El proceso de concentración de las cajas de ahorro puede dificultar la ampliación de los depósitos, teniendo en cuenta el alcance geográfico limitado y reducir las operaciones en las localidades en las que la institución actúa. Se destaca la mayor dificultad en el mantenimiento de los compromisos de la "Obra Social", lo que se refleja en el volumen de puestos de trabajo, en la dinámica social y financiera de las localidades. Es importante señalar que las cajas atendían cerca de 96\%

era estimular el desarrollo del sector privado. Cualquier entidad puede seguir su proceso de reestructuración, y, para los que tienen dudas sobre su viabilidad tendría treinta días para presentar al Banco de España un "plan de viabilidad", que contiene los posibles escenarios para al menos los próximos tres años. La recapitalización se daría principalmente por las fusiones y adquisiciones, con medidas disciplinarias rígidas para las instituciones involucradas (VALVERDE; MAUDOS, 2011).

Con respecto a la Ley de Cajas, que la reestructuración está aún en curso, algunas consideraciones son relevantes en vista de las consecuencias a nivel regional. Esta ley tiene por finalidad facilitar la expedición de activos financieros en los mercados de capitales, ampliando su financiación. Esta ley también promueve la reducción de la interferencia política en la administración de estas entidades y somete a estas instituciones a una mayor presentación de la disciplina de mercado. En resumen, es evidente que tales medidas tienen por objeto poner fin a facilitar la entrada de capital privado en las cajas a través de las siguientes medidas: (i) la creación de un Sistema Institucional de Protección (SIP), en la que las cajas puede fusionarse con un banco que actúa como la entidad central, mutualizando sus beneficios y distribuyendo su solvencia, (ii) la transferencia de la parte financiera de la caja a un banco que detenga al menos 50\% de su propiedad, y, finalmente (iii) la transformación de la caja en una fundación que tiene como propietario un banco que le transfiera toda las operaciones bancarias. 
del territorio español con sus servicios, ya que entre sus principales objetivos era reducir la exclusión financiera Ayade, Schmidt y Valverde (2010). Estas instituciones, más especializada en atender al comercio minorista, tiene una actuación más directa en las áreas económicamente deprimidas y menos pobladas, desarrollando productos específicos para las familias, las empresas pequeñas y medianas así como a los servicios de microfinanzas.

Como factores agravantes de este escenario general, se presentan la débil y aún incierta recuperación de la economía española lo que afecta a los resultados de los bancos con respecto a la creciente morosidad, la difícil recuperación de los precios de los activos inmobiliarios, la alta concentración del riesgo en el sector inmobiliario, el aumento de la preferencia por la liquidez de los agentes y de los bancos así como el mayor racionamiento del crédito a las regiones más vulnerables.

Teniendo en cuenta que este proceso de reestructuración bancaria aún está en curso, es difícil saber al cierto las consecuencias concretas. Solamente en el largo plazo los efectos de las interferencias y de la concentración bancaria podrán ser efectivamente analizados.

\section{Análisis Exploratorio de los Indicadores Bancarios}

Para el análisis exploratorio de este artículo, se ha cogido a datos de las instituciones oficiales de los dos países. En el caso brasileño, los datos son del Banco Central $^{5}$ y el Instituto Brasileño de Geografía y Estadística. En el caso español, del Banco de España y el Instituto Nacional de Estadística (INE).

Resulta importante subrayar la dificultad de obtener datos financieros en el nivel de desagregación regional requerida para este trabajo, sobre todo para la España. De parte del Banco de España, los datos disponibles se refieren a las rubricas de crédito y depósitos consolidados (este último obtenido por la suma de los depósitos a la vista, depósitos a plazo y de ahorro) realizados por comunidad autónoma. Tal dificultad limita el análisis más detallada de las estrategias bancarias regionales, así como inviabiliza la construcción de un mayor número de indicadores. Respecto

5 Datos de las Estadísticas Bancarias por Municipios (ESTBAN), del Banco Central de Brasil elaborado por el Laboratorio de Estudios de Moneda y Territorio (LEMTe/CEDEPLAR). Esta fuente presenta datos mensuales de sucursales bancarias para 3.465 municipios del país, a partir de la década de 2000. Para la comparación intertemporal se consideraron los años 2004, 2006, 2008 y 2012. Respecto a los datos de España, el análisis se inició a partir del de tres años: 2007, 2009, 2012. Como ya se mencionó, en el período 2004-2007, la tasa de crecimiento del crédito eran altos. Desde entonces, la situación del mercado de crédito en España ha cambiado sustancialmente, y se observa una fuerte caída en el volumen de operaciones. La elección del año 2009, por lo tanto, refleja los efectos de esta inversión de crecimiento del crédito y enfriar el crecimiento de la economía española con el impacto de la crisis financiera. En 2012, los datos deben revelar las consecuencias más actuales de los cambios realizados en el proceso de reestructuración bancaria española. Las opciones en estos años se refieren a los períodos de auge en el ciclo de crecimiento para ambos países, alternando con períodos de inestabilidad. 
a los indicadores presentados a continuación, estos tienen como objetivo proporcionar información sobre los efectos de la crisis en estos mercados:

a) Indicadores de Market-Share e de Porte Bancario: el objetivo de ambos es captar la participación de las instituciones bancarias en la provisión de los recursos, respecto a la participación relativa que cada región tiene el sistema bancario nacional. Se calcula a partir del Índice de HerfindallHirschman, ampliamente utilizado en la literatura, lo que constituye una primera medida de la capacidad de las firmas bancarias en ejercer su poder de mercado. Para la construcción de este indicador ha sido utilizado la participación de cada institución en el total de depósitos domésticos, a partir de los datos de ESTBAN (Brasil). ${ }^{6}$ Para el caso de España, fue utilizada la participación de la Comunidad Autónoma en la suma total anual de los depósitos del Gobierno y otros sectores residenciales (OSR);

b) Coeficiente regional de crédito: representa la participación relativa de los préstamos de un Estado frente al volumen total de préstamos del país y la participación del PIB del Estado en el PIB total. Si esta relación es menor que 1 se puede interpretar que el crédito a la región no está en consonancia con su contribución a la economía del país (vice y versa);

c) Indicador de la Preferencia por la Liquidez (PPL) ${ }^{7}$ (CROCCO; CAVALCANTE; BARRA, 2005): Total de depósitos a la vista sobre los préstamos totales. Este indicador busca capturar un mayor o menor disposición de los bancos a permanecer más o menos líquido, lo que influye en la decisión de prestar. Se espera que en las regiones periféricas, el indicador de preferencia por la liquidez tiene un valor mayor, lo que significa que los bancos son menos disponibles a ofertar recursos.

\subsection{Análisis Exploratorio de los Indicadores - Brasil}

Los indicadores descritos anteriormente están representados por las figuras siguientes, con el objetivo de caracterizar el mapa bancario brasileño antes y después de la última crisis financiera. Estos indicadores reflejan el peso del crédito y el sistema bancario de ambos países. En primer lugar, se tiene el indicador de porte bancario.

$6 \quad$ Estadísticas bancarias mensuales. Base de datos publicados por el Banco Central de Brasil, que comprenden el balance de las cuentas de los bancos en todo el país agrupados por estados federativos.

7 Este indicador se muestra adecuado para el análisis en el ámbito de las agencias, teniendo en cuenta que en las regiones periféricas, los bancos no tienen una cantidad representativa de activos financieros, mientras que en las centrales existe una alta densidad de ellos. Para efecto de la comparación regionalizada se hace la opción de utilizar este indicador. 
Figura 1 - Indicador del porte bancario - Brasil

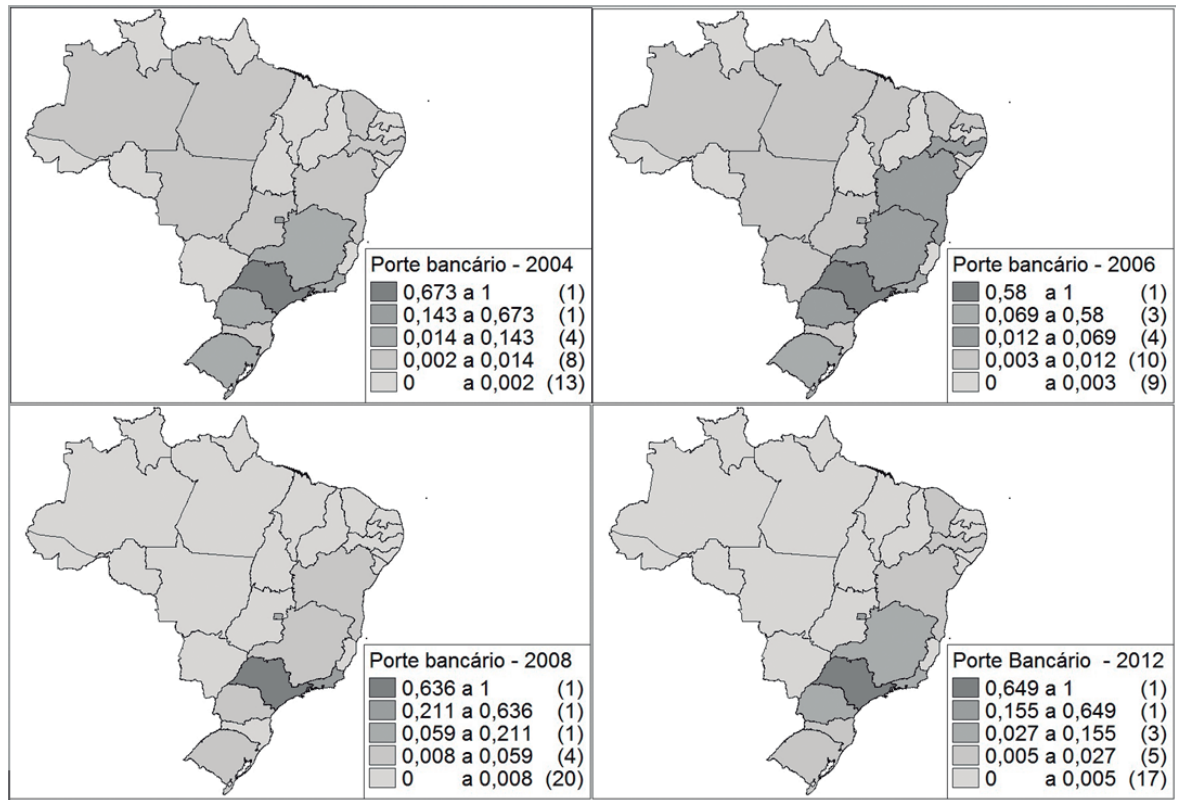

Fuente: LEMTe/UFMG a partir de los datos de ESTBAN/BCB.

El mapa muestra un claro patrón del sistema bancario brasileño: el peso del sistema bancario es relativamente mayor en las regiones más desarrolladas, más precisamente, en São Paulo, comparado a las otras regiones. Este estado es el principal centro dinámico brasileño y centraliza las operaciones del sistema financiero. Esto explica tanto el indicador y el tamaño del banco cuanto al cociente Regional de Crédito. Según Crocco (2010), históricamente, las regiones más desarrolladas concentran las sedes y filiales de firmas industriales, principalmente de participación extranjera. La mayor participación del capital nacional se daba en actividades más intensivas en trabajo, fuertemente sujetas a mayor presión de la competencia. Muchas de estas firmas establecerán sucursales e plantas de producción en regiones menos desarrolladas del país, favorecidas por incentivos fiscales. Todavía los headquarters de estas firmas, en el punto de vista industrial y financiero continúan ubicados en las regiones centrales (principalmente en la región Sudeste de Brasil).

Las regiones más desarrolladas se benefician de una amplia gama de depósitos y recursos destinados a otras aplicaciones centradas en su sede corporativa y de mayor concentración de crédito. Como los depósitos a la vista son la única fuente de recursos gestionados por la agencia, esto explicaría por qué las regiones menos dinámicas (con mayor preferencia por la liquidez), serían menos propensas a prestar debido al riesgo de convertirse en iliquidez (lo que repercute en el menor 
número de las líneas de financiación y racionamiento de recursos para estas regiones). El mayor deterioro de los depósitos en las regiones Norte y Nordeste también se puede explicar por el hecho de que estas regiones son menos diversificadas sobre el punto de vista financiero y, en general, de baja renta, lo que hace con que la base captación de los depósitos sea más restricta.

La concentración de los recursos financieros en las regiones del centro es también el resultado de las estrategias bancarias territorialmente distintas, influenciados por clara preferencia por la liquidez entre las regiones. En las regiones menos desarrolladas, debido a la baja dinámica económica local, los bancos están menos dispuestos a ofertar crédito, lo que resulta en una concentración de las operaciones en las regiones más desarrolladas. Esto puede ser visto en el gráfico Cociente Regional de Crédito (QRC).

Figura 2 - Coeficiente regional del crédito - Brasil

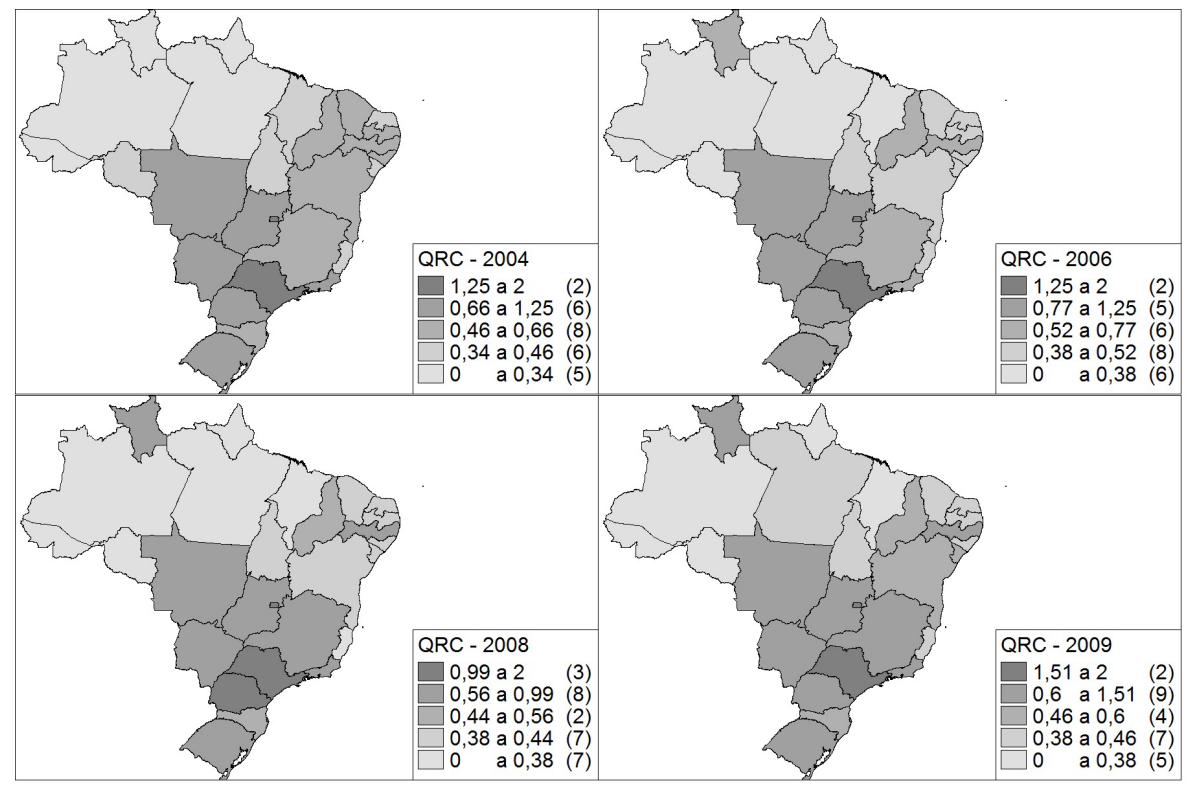

Fuente: LEMTe/UFMG a partir de los datos de ESTBAN/BCB y IBGE.

Través de las figuras se percebe que en después de la crisis financiera, se ha mantenido la concentración bancaria al Sudeste de Brasil. A partir de la presentación de los distintos años, se percebe el efecto procíclico de las instituciones bancarias. En los años anteriores a la crisis, la participación del crédito en las regiones menos desarrolladas ha coincidido con la proporción de la que tenía el PIB nacional. Con el inicio de la crisis financiera global y por efecto de la desaceleración económica - sobre todo en las regiones periféricas - la caída en la oferta de crédito en estas áreas fue relativamente mayor respecto al producto. 
Durante los años estudiados, los estados de las regiones Norte y Nordeste presentaron una reducida participación relativa de la distribución del crédito como contribución al PIB. Situación contraria se observa para los estados del Sudeste y Medio Oeste. Estos hechos sostienen una vez más que la concentración del crédito es resultado de las estrategias bancarias territorialmente diferenciadas, influenciadas por la preferencia por la liquidez principalmente bancaria. En los estados menos desarrollados, debido a la economía local tener un menor dinamismo, los bancos están menos dispuestos a conceder nuevas líneas y ampliar el volumen de crédito. En el contexto posterior a la crisis, esta tendencia se mantiene.

Los dos indicadores vistos hasta el momento muestran, en primer lugar, que la mala asignación de los créditos se relaciona con la distribución desigual de la riqueza del país así como el hecho de lo que la evolución de la participación del sector bancario en una región sigue la distribución regional del PIB.

Figura 3 - Indicador de la preferencia por la liquidez - Brasil

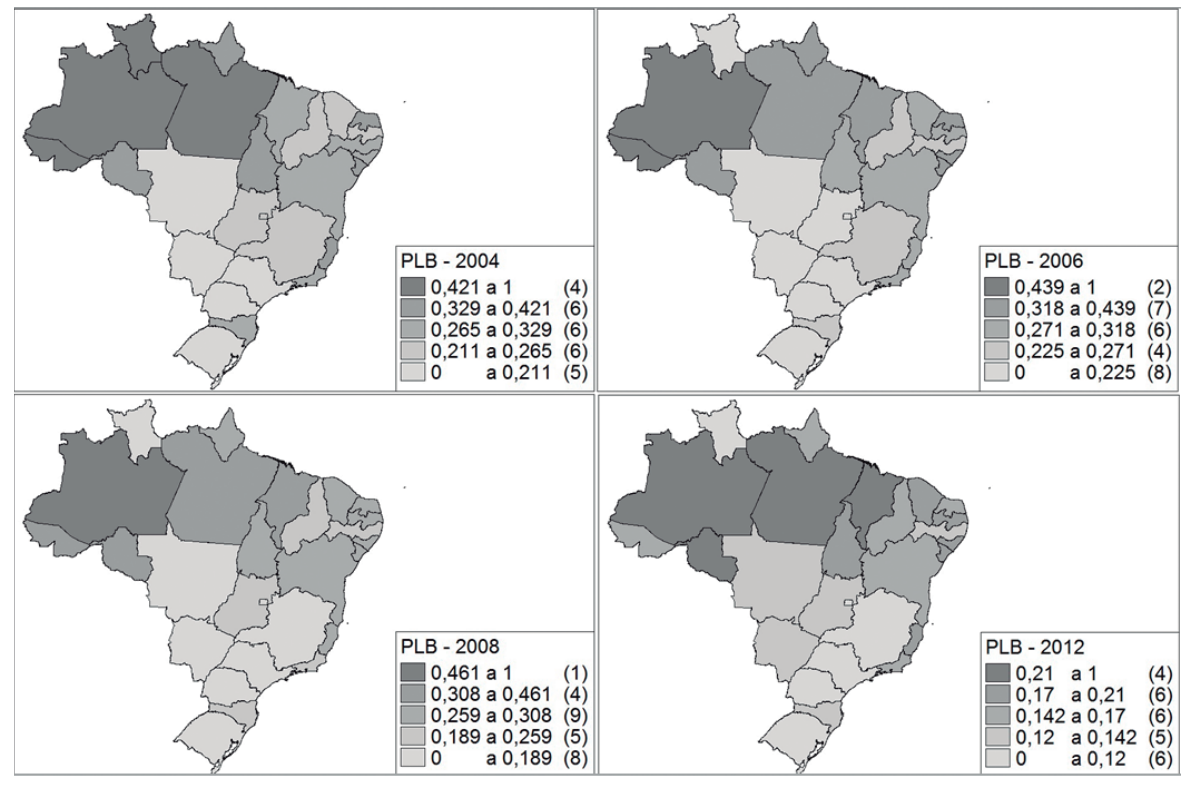

Fuente: LEMTe/UFMG a partir de los datos de ESTBAN/BCB.

El indicador de preferencia por la liquidez de las sucursales bancarias (PLB) mide la disposición de las mismas en conceder crédito y ponerse menos liquida. Las cifras a continuación confirman la hipótesis de Dow (1993), según la cual la preferencia por la liquidez tiende a ser mayor en las regiones menos desarrolladas. En otras regiones, los bancos muestran una mayor disposición a prestar, sobre todo en períodos de boom del ciclo económico. 
Tanto una mayor diversificación de la estructura productiva (y de las oportunidades de negocio), como el nivel de ingreso más alto (asociado con una menor preferencia de liquidez del público) ayudan a explicar el comportamiento de los bancos y justifica por qué la región Norte presenta mayor preferencia por la liquidez (y la región Sudeste, la más baja). Se observa que posteriormente a la crisis financiera se eleva la preferencia por la liquidez en la región Medio Oeste debido al comportamiento defensivo de los bancos frente a una región ampliamente dependiente de los préstamos bancarios (especialmente para la agricultura y otras actividades de exportación).

\subsection{Análisis Exploratorio de los Indicadores - España}

El indicador de la cuota de mercado de la banca española refleja la preponderancia de los principales centros financieros del país (Madrid) y de la banca (Cataluña, País Vasco y Comunidad Valenciana) como regiones de mayor presencia de las entidades (en particular, los grandes bancos comerciales), seguido de las cajas de Ahorro y Cooperativas.

La comunidad autónoma de Andalucía presenta alto market share lo que puede ser explicado por la presencia de un grande número de cajas de ahorro, por lo menos hasta la crisis. Andalucía y la Comunidad Valenciana, debido al peso del sector inmobiliario, fueron muy afectadas por el colapso de la vivienda, la reducción de la base de financiamiento y de depósitos. Tal dinámica fue acentuada porque una parte de las cajas incurrieron en problemas de liquidez, sofriendo la intervención por parte del Banco de España. Los efectos de las fusiones e intervenciones aún son analizados, pero se espera que después de consolidado el proceso de reestructuración bancaria, se aumente la concentración bancaria de estas regiones. 
Figura 4 - Indicador del market share - España

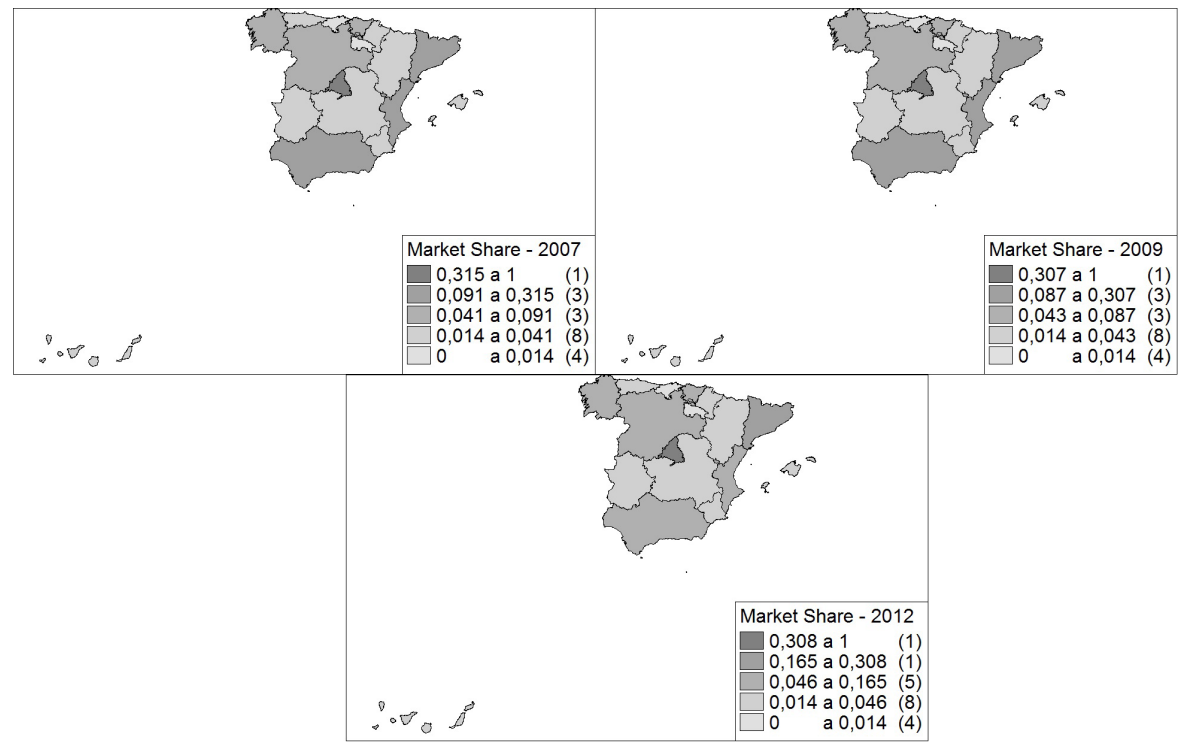

Fuente: LEMTe/UFMG a partir de los datos de Banco de España.

Las regiones que presentaron los más bajos resultados para la captación de depósitos), niveles de ingresos y dinamismo económico (por ejemplo, Extremadura, Navarra, Castilla La Mancha, Aragón), o son regiones muy especializadas o muy pequeñas desde el punto de vista de la población (La Rioja, Murcia, Baleares y Canarias). A seguir, otro indicador importante a analizar es lo de preferencia por la liquidez. 
Figura 5 - Indicador de la preferencia por la liquidez - España

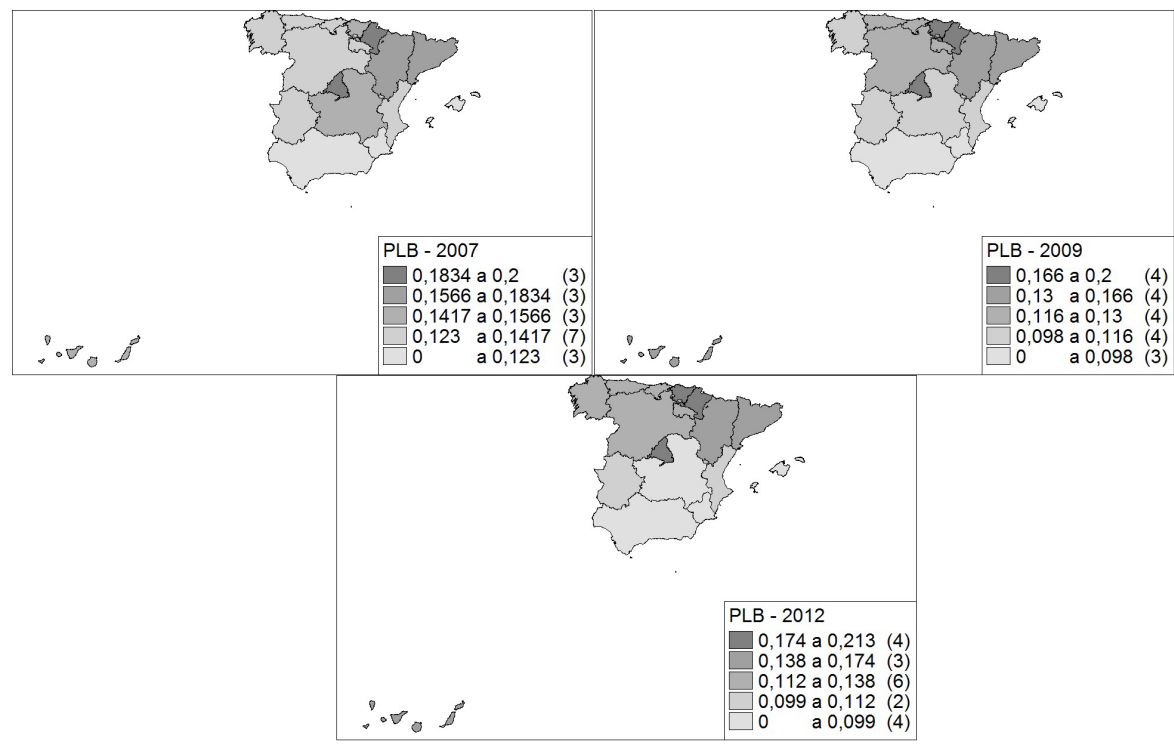

Fuente: LEMTe/UFMG a partir de los datos de Banco de España.

Como ha sido mencionado, este indicador refleja la disposición de los bancos de conceder crédito a la región, de acuerdo con sus expectativas. Por el lado de la demanda de crédito, preferencia por la liquidez del público afectará a sus respectivas decisiones de cartera. Cuanto mayor es la preferencia por la liquidez, más recogen a las posiciones en activos líquidos y reducen la oferta de crédito.

Contrariamente a lo que se había esperado, las regiones con mayor concentración financiera, Madrid y el País Vasco (sede del Grupo Bilbao Vizcaya), mostraron un alto nivel de preferencia por la liquidez a partir de 2009, también seguida de Navarra. Esto puede estar reflejando la recesión por la que pasa la economía española, lo que limita el volumen de los depósitos a la vista. Además, la fuerte contracción del crédito y las medidas de austeridad anunciadas por el Banco de España, sumadas al proceso de reestructuración bancaria, tienden a reducir depósitos a la vista o dirigirlos a otras aplicaciones.

La baja preferencia por la liquidez en algunas regiones periféricas refuerza la importancia de los bancos locales al reducir los problemas de acceso a los servicios financieros y la provisión de crédito, incluso en las comunidades autónomas más remotas. Por último, en relación con el crédito coeficiente regional, es evidente que los principales centros financieros (Madrid, Cataluña) siguen teniendo una mayor participación del crédito en relación con su contribución al PIB comparado a otras regiones. 
Figura 6 - Coeficiente regional del crédito - España

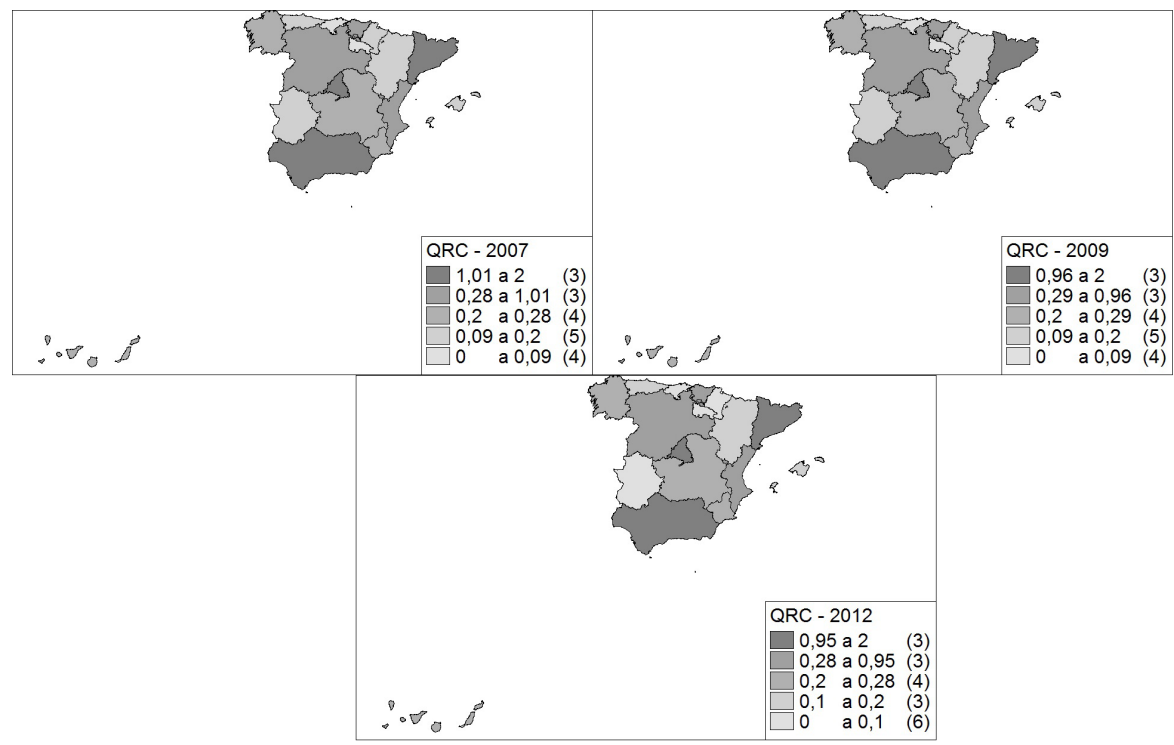

Fuente: LEMTe/UFMG a partir de los datos de Banco de España.

Tal evidencia refuerza la concentración del crédito como también es resultado de las estrategias bancarias diferenciadas territorialmente, influenciadas por diferentes características económicas de las regiones. En las regiones menos desarrolladas, debido a la economía local presentar una dinámica económica más letárgica, los bancos están menos dispuestos a ofertar crédito, lo que acentúa la concentración de las operaciones en las regiones más desarrolladas. Posteriormente a la última crisis financiera, se ha verificado un mayor número de comunidades autónomas con bajo coeficiente regional de crédito.

\section{Consideraciones Finales}

Este estudio ha buscado ofrecer un análisis comparativo de los efectos de la reciente crisis financiera en los sistemas bancarios brasileños y españoles desde una perspectiva regionalizada. Ambos los países han conformado su desarrollo económico a través del sistema bancario. Una vez presentadas las caracterizaciones recientes y el impacto de la última crisis financiera en ambos países, se ha pasado a un análisis de los indicadores regionales.

En Brasil, se ha encontrado la misma evidencia de las estrategias bancarias vigentes en favor de la concentración y de la búsqueda por rentabilidad en las regiones más desarrolladas sobre el punto de vista económico. La reciente crisis financiera ha acentuado este patrón. La especificidad del caso brasileño está en 
el papel de los bancos públicos, importantes para mantener el carácter funcional, en particular para las regiones menos desarrolladas (principalmente porque la presencia de los bancos regionales es limitada).

Por lo tanto, hay una necesidad de revisar la rigidez de la aplicación de las normas de Basilea III $^{8}$ de modo especial a las instituciones financieras públicas y a los bancos de desarrollo de Brasil (o incluso en los bancos regionales, como en el caso de España). Tales instituciones no deben ser clasificadas con el mismo rigor de las normas de Gestión del Riesgo, como están sometidas las otras Instituciones Financieras, debiendo ser más flexible la aplicación de las normas reglamentarias. La generalización y la falta de adecuación específica para estas instituciones más "funcionales", puede comprometer la actuación de las mismas en la recuperación económica como también en el desarrollo productivo futuro, especialmente en las regiones más afectadas por la crisis.

En línea con el enfoque de Martins (2012), para el caso de los bancos de desarrollo brasileño y ampliando el argumento a favor de los bancos regionales de España, se subraya la importancia de estas instituciones con respecto a otras instituciones financieras, principalmente porque se están articuladas con las políticas de desarrollo elegidos por el Estado con el sentido de promover el desarrollo económico local. En sentido contrario, los bancos privados tienen sus objetivos guiados por el lucro de corto plazo y la cotización a los accionistas, dejando de apoyar a ciertos segmentos de mercado considerados más arriesgados.

Los espacios económicos marginales conviven en un ambiente de gran incertidumbre para las instituciones financieras. Independientemente del margen de riesgo que se puede aplicar en estas regiones, hay una mayor restricción al crédito privado. En este sentido, los bancos públicos regionales deben actuar en estos segmentos. Para se concretar esta actuación es necesario que exista estrategias de gestión de riesgos diferenciadas, lo que ha sido desconsiderado por las reglas de prudencia y regulación.

En el caso español, la segmentación del mercado con la presencia de los bancos regionales debería favorecer la mitigación de los efectos de la crisis, por permitir mayor integración entre la banca y el desarrollo regional, debido a la presencia de bancos locales (cajas de ahorro) y las cooperativas. Estas instituciones son esenciales desde el punto de vista "funcional", teniendo en cuenta la gran importancia de las pequeñas y medianas empresas y el sector agrícola al tejido económico español. Sin embargo, la reciente crisis financiera ha hecho con que el Banco de

$8 \quad$ El acuerdo de Basilea III tiene medidas generales para aumentar los requisitos de capital con el fin de incorporar los riesgos de la banca, la introducción de un colchón de seguridad en el cumplimiento de las medidas de capital y de solvencia de capital anticíclico, la mejora en el capital regulatorio a favor de la core capital, la introducción de un coeficiente de apalancamiento y liquidez y la expansión de los instrumentos de control para ser utilizado por el supervisor (Comunicado 020 615/17 Febrero 2011/Bacen). 
España incentive el proceso de reestructuración bancaria por medio de las fusiones frías o de otras formas de intervención. Se ha noticiado que muchos bancos regionales fueron comprados por grandes bancos, lo que amplía la concentración bancaria y altera el mapa bancario español. La concentración del mercado ha aumentado, lo que tiende a reducir el crédito a las regiones periféricas, fortaleciendo las desigualdades regionales. Las consecuencias de este proceso en curso todavía no son claras, así como sus impactos regionales. Pero se puede inferir que el carácter funcional del sistema bancario en las comunidades autónomas será afectado.

La crisis financiera instalada en España no se puede resolver solamente con la reestructuración y reorganización del sector bancario. Es incuestionable que el crecimiento económico depende de la recuperación del mercado de crédito. Sin embargo, para que este crecimiento sea sustentable es necesario más do que mercado de crédito eficientes y activos, es necesario que se rescaten los proyectos empresariales comprometidos con el crecimiento y el desarrollo económico, como también de la revisión de los objetivos de austeridad fiscal.

La economía española ha pasado por una fase de rescate de su credibilidad a través de este proceso de reestructuración bancaria, proceso conducido por el mercado, los organismos multilaterales y las instituciones financieras, monitoreados por el BCE y el Banco de España. Sin embargo, el aumento del control reglamentario y la reducción del número de entidades pueden obstaculizar la oferta de recursos a las regiones menos desarrolladas. Es necesario rescatar los planes de negocios y créditos direccionados al sector inmobiliario como también a otras actividades productivas, a las pequeñas y medianas empresas. Se trata de una agenda de investigación que se está abierta y presenta avances muy recientes, cuyos efectos sólo podrán ser analizados de modo más completo en el medio y largo plazo.

\section{Bibliografía}

AYADI, R.; SCHMIDT, R. H.; VALVERDE, S. C. Investigating diversity in the banking sector in Europe: the performance and role of saving banks. Brussels: Centre for European Policy Studies, 2010.

BANCO CENTRAL DO BRASIL. Comunicado n. 020615. Brasília, DF: BCB, 2011. Disponible en: https: <www3.bcb.gov.br/normativo/detalharNormativo.do?method=detalharNormati voE $\mathrm{N}=111011733$ ? > . Acceso en: 17 jan. 2012.

. Estadísticas Bancarias por Municipios (ESTBAN). 2012. Brasília. Disponible en: <http://www4.bcb.gov.br/fis/cosif/estban.asp>. Acceso en: 02 jan. 2012.

. Relatório de Moeda e Crédito. Brasília, DF: BCB, 2010. Disponible en: <http://www. bcb.gov.br/Pec/Depep/Spread/REBC2010.pdf> . Acceso en: 20 sept. 2012. 
BANCO DE ESPAÑA. Boletim Estatístico do Banco de España. Varios años. Disponible en: <http:www.bde.es>. Acceso en: 12 ago. 2012.

. Cuentas financieras de la economía española. 2012. Disponible en: <http:www.bde.es $>$. Acceso en: 15 sept. 2012.

. Evolución y reforma de las cajas de ahorros. 2011. Disponible en: <http://www.bde. es/webbde/es/secciones/prensa/reestructura_sane/ficheros/notareformacajas210211.pdf >. Acceso en: 15 sept. 2012.

BARBOSA, N. Latin America: counter-cyclical policy in Brazil: 2008-09. Journal of Globalization and Development, v. 1, n. 1, p. 1-12, 2010.

BASEL COMMITTEE. Basel III: a global regulatory framework for more resilient banks and banking systems. Basel: Bank for International Settlements, 2010a.

. Basel III: international framework for liquidity risk measurement, standards and monitoring. Basel: Bank for International Settlements, $2010 \mathrm{~b}$.

CARVALHO, F. C. Sobre a preferência pela liquidez dos bancos. In PAULA, L. F.; OREIRO, J. L. (Org.). Sistema financeiro: uma análise do setor bancário brasileiro. Rio de Janeiro: Campus; Elsevier, 2007.

CROCCO, M.; FIGUEIREDO, A. T. L.; SANTOS, F. Differentiated banking strategies across the territory: an exploratory analysis. Journal of Post Keynesian Economics, v. 33, n. 1, p. 127$150,2010$.

CROCCO, M. Moeda e desenvolvimento regional e urbano: uma leitura Keynesiana e sua aplicação ao caso brasileiro. 2010. 172 f. Tesis (Profesor Titular) - Departamento de Ciencias Econômicas, Universidad Federal de Minas Gerais, Belo Horizonte, 2010.

CROCCO, M.; CAVALCANTE, A.; BARRA, C. The behavior of liquidity preference of banks and public and regional development: the case of Brazil. Journal of Post Keynesian Economics, v. 28, n. 2, p. 217-40, Dec./Jan. 2005.

DOW, S. The stages of banking development and the spatial evolution of financial systems. In: MARTIN, R. (Ed.). Money and the Space Economy. London: Wiley, 1999.

DOW, S. Money and the economic process. Aldershot: Edward Elgar, number 147, April.1993.

FREITAS, M. C. P. Os efeitos da crise global no Brasil: aversão ao risco e preferência pela liquidez no mercado de crédito. Estudos Avançados, v. 23, n. 66, p. 125-145, 2009.

HERMANN, J.; PAULA, L. F. Economic development and the functionality of the financial system in Brazil: a Keynesian approach. In: ENCONTRO INTERNACIONAL DA ASSOCIAÇÃO KEYNESIANA BRASILEIRA, 4., 2011, Rio de Janeiro. Anais... Rio de Janeiro: $\mathrm{AKB}, 2011$.

INTERNATIONAL MONETARY FUND. Spain: the reform of Spanish savings banks technical notes. Washington, D.C.: IMF, May 2012. (IMF Staff Country Reports, n. 12/141). 
Spain: financial sector assessment program: Technical note: regulation, supervision, and governance of the Spanish cajas. Washington, D.C.: IMF, June 2006. (IMF Staff Country Reports, n. 06/2015).

KREGEL, J. The global crisis and the implications for developing countries and the BRICs: Is the "B" really justified? Revista de Economia Política, v. 29, n. 4, p. 341-356, Oct./Dec. 2009.

MARTINS, N. M. Basiléia III: novas medidas, velhos problemas. In: ENCONTRO INTERNACIONA DA ASSOCIAÇÃO KEYNESIANA BRASILEIRA, 3., 2012, Rio de Janeiro. Anais... Rio de Janeiro: AKB, 2012.

REZENDE, L. P. F. Inovação tecnológica e a funcionalidade do sistema financeiro - uma análise do balanço patrimonial dos bancos no Brasil. 2012. 176 f. Tese (Doutorado em Economia) - Departamento de Ciencias Econômicas, Centro de Desenvolvimento e Planejamento Regional, Universidad Federal de Minas Gerais, Belo Horizonte, 2012.

RODRIGUEZ-FUENTES, C. J., SILVA, F. F. Menos "arquitetura" financeira e mais banco "funcional”. In: MENDONÇA, A. R. R. et al. (Org.). Dossiê da Crise II. Campinas: Associação Keynesiana Brasileira, 2010. p. 48-52.

RODRIGUEZ-FUENTES, C. J.; MARRERO, P. D.; HERRERA, A. O. Integración monetaria y segmentación regional de mercados bancarios. Revista de Estudios Regionale, n. 70, p. 41-61, mayo-ago. 2004.

RODRIGUEZ-FUENTES, C.; DOW, S. Financial deregulation, banking competition and regional development: the Spanish experience.In: CONGRESS EUROPEAN REGIONAL SCIENCE ASSOCIATION, 39., 1999, Dublin. Anais... Dublin: Ersa, 1999. p. 23-27.

SILVA, F. F. Centralidade e impactos regionais de política monetária: um estudo dos casos brasileiro e espanhol. 2011. 293 f. Tese (Doutorado em Economia) - Departamento de Ciencias Econômicas, Centro de Desenvolvimento e Planejamento Regional, Universidad Federal de Minas Gerais, Belo Horizonte, 2011.

STUDART, R. Investment finance in economic development. London; New York: Routledge, 1995.

VALVERDE, S. C.; MAUDOS, J. Reflexiones en torno a la reestructuración del sector bancario español. Cuadernos de Información Económica, n. 221, p. 81-95, mar. /abr. 2011.

VALVERDE, S. C.; HUMPHREY, D.; FERNÁNDEZ, F. R. Deregulation, bank competition and regional growth. Regional Studies, v. 37, n. 3, p. 227-237, May 2003.

Recebido em: 25/05/2014. Aceito em: 11/09/2014. 